\title{
Article \\ Comparative Efficacy of Various Stents for Palliation in Patients with Malignant Extrahepatic Biliary Obstruction: A Systematic Review and Network Meta-Analysis
}

\author{
Chan Hyuk Park ${ }^{1}$, Se Woo Park ${ }^{2, * \mathbb{C}}$, Jang Han Jung ${ }^{2}$, Eun Suk Jung ${ }^{2}{ }^{\oplus}$, Jung Hee Kim ${ }^{2}$ and Da Hae Park ${ }^{2}$ \\ 1 Department of Internal Medicine, Hanyang University Guri Hospital, Hanyang University College of \\ Medicine, Guri 11923, Korea; yesable7@gmail.com \\ 2 Division of Gastroenterology, Department of Internal Medicine, Hallym University Dongtan Sacred Heart \\ Hospital, Hallym University College of Medicine, Hwaseong 18450, Korea; con2000@hallym.or.kr (J.H.J.); \\ esjung@hallym.or.kr (E.S.J.); jungheekim@hallym.or.kr (J.H.K.); dahaepark82@gmail.com (D.H.P.) \\ * Correspondence: mdsewoopark@gmail.com; Tel.: +82-31-8086-2858
}

Citation: Park, C.H.; Park, S.W.; Jung, J.H.; Jung, E.S.; Kim, J.H.; Park, D.H. Comparative Efficacy of Various Stents for Palliation in Patients with Malignant Extrahepatic Biliary Obstruction: A Systematic Review and Network Meta-Analysis. J. Pers. Med. 2021, 11, 86. https://doi.org/ 10.3390/jpm11020086

Academic Editor: Ivo Boškoski Received: 28 December 2020

Accepted: 27 January 2021

Published: 30 January 2021

Publisher's Note: MDPI stays neutral with regard to jurisdictional claims in published maps and institutional affiliations.

Copyright: (c) 2021 by the authors. Licensee MDPI, Basel, Switzerland. This article is an open access article distributed under the terms and conditions of the Creative Commons Attribution (CC BY) license (https:// creativecommons.org/licenses/by/ $4.0 /)$.

\begin{abstract}
Although many studies have investigated the efficacy of stent placement for patients with malignant extrahepatic biliary obstruction, the clinical outcomes and adverse events of biliary stenting have not been comprehensively evaluated. We searched all relevant randomized-controlled trials that evaluated the comparative efficacy of biliary stents, including the plastic stents, uncovered self-expandable metal stents (SEMSs), and covered SEMSs in patients with malignant extrahepatic biliary obstructions. Twenty-one studies with 2326 patients were included. Both uncovered and covered SEMSs had a lower risk of recurrent biliary obstruction (RBO) compared to plastic stents (risk ratio (RR) (95\% confidence interval [CI]): uncovered vs. plastic, $0.46(0.35-0.62)$; covered vs. plastic, $0.46(0.34-0.62))$. A comparison of the groups using SEMSs revealed that tumor ingrowth was common in the uncovered SEMS group, while stent migration, tumor overgrowth, and occlusion by sludge were common in the covered SEMS group; however, the overall risk of RBO did not differ between these groups (RR (95\% CI): uncovered vs. covered: $1.02(0.80-1.30)$ ). Although the main causes of RBO vary across stents, RBO risk was similar between uncovered and covered SEMS groups. Both SEMSs have superior efficacy in terms of RBO compared to plastic stents.
\end{abstract}

Keywords: malignant biliary obstruction; stent; plastic stent; metal stent; recurrent biliary obstruction

\section{Introduction}

Appropriate biliary drainage is important for relieving symptoms and extending life expectancy in patients with unresectable malignant biliary obstruction (MBO) [1]. Biliary drainage techniques involving endoscopic retrograde cholangiopancreatography (ERCP) or percutaneous transhepatic biliary drainage were established approximately 40 years ago [2,3]. In the 1980s, when endoscopic drainage was first introduced, plastic stents were typically used for the procedure [4]. Biliary drainage using plastic stents was as effective as surgical drainage [2,4]. In the 1990s, self-expandable metal stents (SEMSs) were introduced, and their efficacies were compared with those of plastic stents in many studies [5]. Endoscopic drainage using SEMSs prolongs patient survival, lowers adverse events, including recurrent biliary obstruction (RBO) [6], and requires fewer reinterventions compared with drainage using plastic stents [5,7]. Although SEMSs are more expensive than plastic stents, a recent randomized-controlled trial (RCT) showed that the total costs up to one year after stenting did not significantly differ between plastic stent and SEMS groups because of the longer functional time of SEMSs [8].

Regarding subtypes of SEMSs, several meta-analyses compared the efficacies of uncovered and covered SEMSs during endoscopic drainage for patients with extrahepatic MBO $[9,10]$. According to these studies, using covered SEMSs entailed a lower risk of 
tumor ingrowth, but a higher risk of tumor overgrowth, stent migration, and occlusion by sludge or food crap, compared to the use of uncovered SEMSs. RBO, overall adverse events, and the overall survival period did not differ between both SEMSs $[9,10]$.

Although several meta-analyses evaluated differences among the efficacies of stent types, it is still difficult to comprehensively understand this topic as previous meta-analyses focused on pairwise comparisons between two stent types (e.g., plastic vs. metal stents, and uncovered vs. covered SEMSs) that would provide only fragmented comparative efficacies $[5,7,9,10]$. Additionally, without knowing absolute efficacies, it is difficult to know how many patients can avoid stent-related adverse events depending on stent types. Therefore, we performed a network meta-analysis of RCTs comparing efficacies of the different types of stents in patients with extrahepatic MBO. We also investigated the absolute risk of clinical outcomes and stent-related adverse events based on all relevant RCTs.

\section{Materials and Methods}

\subsection{Study Design}

We performed a systematic review and network meta-analysis in adherence to the Preferred Reporting Items for Systematic Reviews and Meta-Analyses statement [11] and the International Society for Pharmacoeconomics and Outcomes Research Task Force on Indirect Treatment Comparisons Good Research Practices report [12].

\subsection{Search Strategy}

All relevant studies published between January 1990 and January 2020 that evaluated the efficacy of stents for palliation in patients with extrahepatic $\mathrm{MBO}$ were retrieved from the MEDLINE, EMBASE, and Cochrane Library databases. The following search string was used: ((bile duct) or (biliary) or (cholangiocarcinoma) or (cholangiocarcinomas) or (pancreatic) or (pancreas)) and ((obstruction) or (obstructive) or (stricture) or (strictures) or (jaundice) or (cholestasis)) and ((nonresectable) or (unresectable) or (palliation) or (palliative) or (palliating) or (inoperable)) and ((stent) or (stents) or (stenting) or (endoprostheses)) and (random*). Appendix ?? shows the detailed search strategies for each database. Additionally, we examined the references of the screened articles to identify additional relevant studies. Our search was last updated at 15 January 2020.

\subsection{Inclusion/Exclusion Criteria}

The inclusion criteria were as follows: (a) population: patients with unresectable extrahepatic $\mathrm{MBO}$, (b) intervention: ERCP with palliative biliary decompression using stents including a plastic, uncovered, or covered stent, (c) comparator: ERCP with palliative biliary decompression with another type of stent, and (d) outcome: technical and clinical success, $\mathrm{RBO}$, and adverse events. Non-human studies, non-original studies, non-RCTs, abstract-only publications, and studies published in languages other than English were excluded.

\subsection{Study Selection}

In the first step of the study selection, duplicated articles, which were retrieved through multiple search engines, were excluded. Thereafter, we examined the titles and abstracts of the articles to exclude irrelevant studies. The full text of the remaining articles was then assessed for eligibility. Two investigators (C.H.P. and S.W.P.) independently evaluated the studies for eligibility and resolved any disagreements through discussion. If an agreement could not be reached, a third investigator (J.H.J.) determined study eligibility. The Cochrane Risk of Bias assessment tool was used for assessing the risk of bias in the included RCTs.

\subsection{Data Extraction and Study Endpoint}

Using a data extraction form developed in advance, two reviewers (C.H.P. and S.W.P.) independently extracted the following information: first author name, year of publication, study design, country, study period, publication language, type of biliary stent (plastic 
stent, uncovered SEMS, or covered SEMS), and clinical outcomes, including clinical success, $\mathrm{RBO}$, and adverse events.

The primary endpoint in this meta-analysis was the comparative efficacy of biliary stents in terms of RBO. Secondary endpoints were causes of RBO, including occlusion by sludge, tumor ingrowth and overgrowth, and stent migration, and stent-related inflammation, including cholangitis, cholecystitis, and pancreatitis.

\subsection{Statistical Analysis}

A direct pairwise meta-analysis was conducted to calculate the risk ratios (RRs) of categorical variables, including those of $\mathrm{RBO}$ and adverse events using a random-effects model. For technical and clinical successes, we calculated crude proportions rather than RRs because the technical or clinical success rates were $100 \%$ in many studies, and those studies could not be included for calculating pooled RRs. Statistical heterogeneity was assessed using two methods: Cochrane's $Q$ test, in which $p$-values of $<0.1$ were considered statistically significant for heterogeneity, and $I^{2}$ statistics, wherein values of $>50 \%$ suggested significant heterogeneity [13]. We assessed publication bias quantitatively using the Begg and Mazumdar adjusted-rank correlation test (publication bias was considered present if $p$ was <0.1) [14]. We also assessed bias qualitatively by inspecting the funnel plots of logarithmic RRs versus their standard errors [15]. When the number of included studies for each pairwise comparison was less than 10, the test for publication bias was not conducted [16]. A direct pairwise meta-analysis was performed using the Review Manager statistical software (version 5.3.5; Cochrane Collaboration, Copenhagen, Denmark) and Comprehensive Meta Analysis (version 2.2.064; Biostat Inc., Englewood, NJ, USA) software.

A frequentist network meta-analysis was performed to calculate direct and indirect estimates and combine mixed estimates [17]. Moreover, each stent type was ranked according to P-scores, which were based solely on the point estimates and standard errors of network estimates [18]. The P-score of each eradication regimen can be interpreted as the mean extent of certainty that a certain regimen was better than another [18]. The network meta-analysis was performed using the R statistical software (version 3.6.2; R Foundation for Statistical Computing, Vienna, Austria) with the netmeta package (version 0.9-1; Rücker et al.). The netmeta package is based on the graph theory methodology used to model the relative treatment effects of multiple treatments under a frequentist framework [19].

\section{Results}

\subsection{Study Selection and Characteristics}

Twenty-one studies including 2326 patients were included in our meta-analysis (Figure 1) [8,20-39].

The baseline patient and lesion characteristics are summarized in Table 1. Studies were published between 1992 and 2020 with an enrollment period that ranged from 1990 to 2018. Six studies evaluated compared the efficacy of plastic stents and that of uncovered SEMSs [20-23,25,37], while three studies compared the efficacy of plastic stents and that of covered SEMSs $[26,30,33]$. The other 11 studies compared the efficacy of uncovered SEMSs and that of covered SEMSs [24,27-29,31,32,34-36,38,39]. The remaining study was a three-arm trial, which compared the efficacy of plastic stents, that of uncovered SEMS, and that of covered SEMS [8]. 

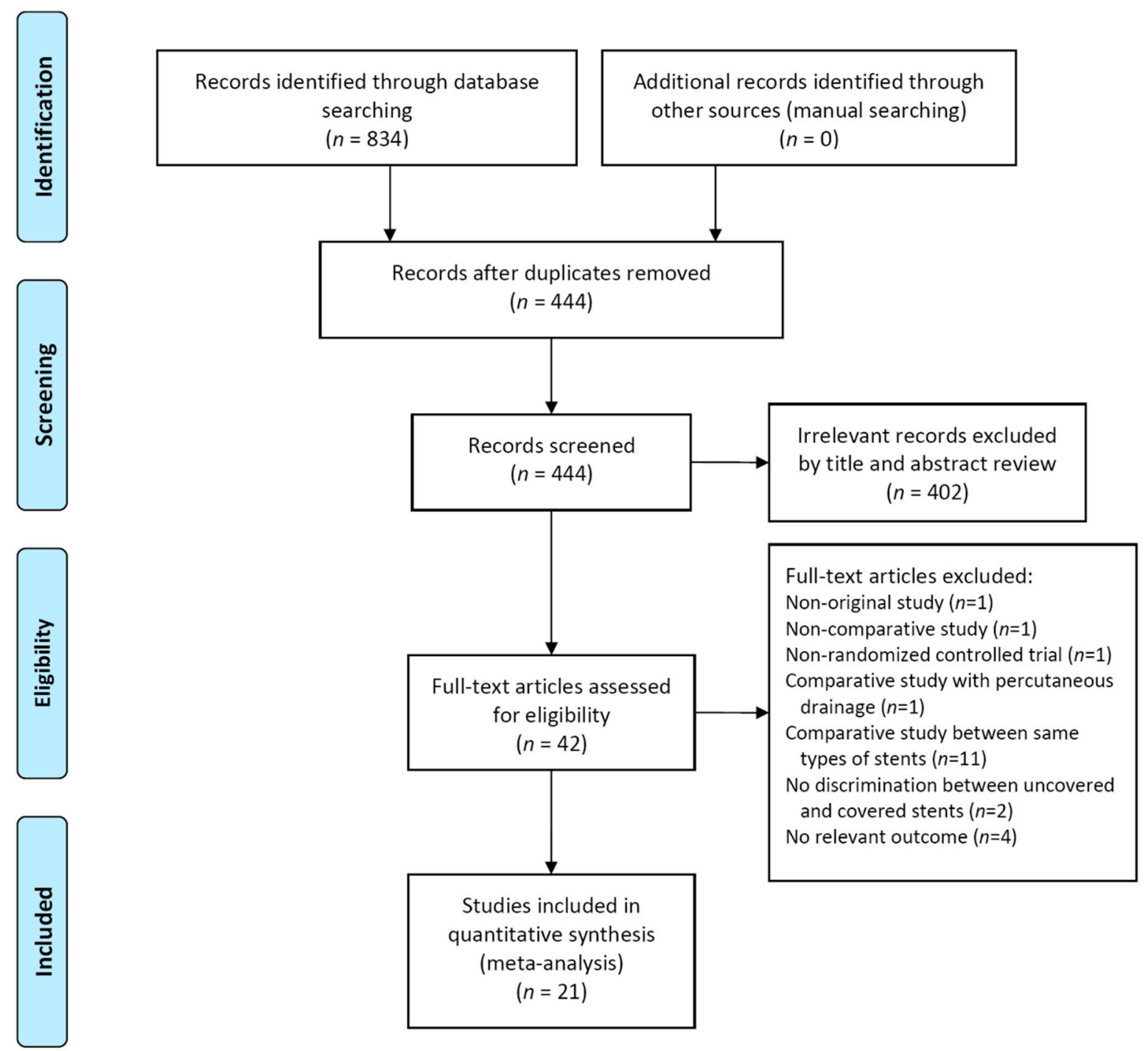

Figure 1. Flow diagram of the studies included in the meta-analysis. 
Table 1. Baseline patient and lesion characteristics in the included studies.

\begin{tabular}{|c|c|c|c|c|c|c|c|c|c|c|}
\hline $\begin{array}{l}\text { First } \\
\text { Author }\end{array}$ & $\begin{array}{l}\text { Publication } \\
\text { Year }\end{array}$ & Country & $\begin{array}{l}\text { Study } \\
\text { Period }\end{array}$ & $\begin{array}{l}\text { Number of } \\
\text { Patients }\end{array}$ & $\begin{array}{c}\text { Age, Year, } \\
\text { Mean } \pm \text { SD }\end{array}$ & Male, n (\%) & $\begin{array}{l}\text { Performance } \\
\text { Status }\end{array}$ & Cause of Obstruction & $\begin{array}{c}\text { Baseline total } \\
\text { Bilirubin, } \\
\text { mg/dL, Mean } \pm \\
\text { SD }\end{array}$ & Used Stent \\
\hline Davids & 1992 & Netherlands & 1990-1992 & $\begin{array}{c}\text { Plastic: } 56 \\
\text { Uncovered: } 49\end{array}$ & $\begin{array}{l}\text { Plastic: } 76.5 \\
\text { (range, 53-96) } \\
\text { Uncovered: } 76 \\
\text { (range, 46-94) }\end{array}$ & $\begin{array}{c}\text { Plastic: } 41.1 \\
\text { Uncovered: } 49.0\end{array}$ & $\mathrm{~N} / \mathrm{A}$ & $\begin{array}{c}\text { Plastic: pancreatic cancer } \\
(89.3 \%), \text { ampullary cancer } \\
(10.7 \%) \\
\text { Uncovered: pancreatic } \\
\text { cancer }(87.8 \%), \text { ampullary } \\
\text { cancer }(12.2 \%)\end{array}$ & $\begin{array}{c}\text { Plastic: median } \\
10.2 \text { (range, } \\
1.1-34.2 \text { ) } \\
\text { Uncovered: } \\
\text { median } 11.3 \\
\text { (range, } 0.7-41.6 \text { ) }\end{array}$ & $\begin{array}{c}\text { Plastic: 10-Fr } \\
\text { endoprostheses (PBN } \\
\text { Medicals, Stenloese, } \\
\text { Denmark) } \\
\text { Uncovered: 30-Fr metal } \\
\text { stent (Wallstent, Schneider, } \\
\text { Switzerland) }\end{array}$ \\
\hline Knyrim & 1993 & Germany & 1990-1992 & $\begin{array}{c}\text { Plastic: } 31 \\
\text { Uncovered: } 31\end{array}$ & $\begin{array}{c}\text { Plastic: } 70.2 \pm \\
1.8 \\
\text { Uncovered: } 70.8 \\
\pm 2.0\end{array}$ & N/A & $\begin{array}{c}\text { ECOG } \\
\text { performance } \\
\text { status } \\
\text { Plastic: median } 3 \\
\text { Uncovered: } \\
\text { median 3 }\end{array}$ & $\begin{array}{c}\text { Plastic: pancreatic cancer } \\
(71.0 \%), \text { bile duct cancer } \\
(3.2 \%) \text {, ampullary cancer } \\
(3.2 \%) \text {, others }(22.6 \%) \\
\text { Uncovered: pancreatic } \\
\text { cancer }(67.7 \%) \text {, bile duct } \\
\text { cancer }(3.2 \%) \text {, ampullary } \\
\text { cancer }(6.5 \%) \text {, others } \\
(22.6 \%)\end{array}$ & $\begin{array}{c}\text { Plastic: median } \\
10 \\
\text { Uncovered: } \\
\text { median } 12\end{array}$ & $\begin{array}{c}\text { Plastic: } 11.5-\mathrm{Fr} \\
\text { polyethylene plastic stent } \\
\text { (Huibregtse biliary stent, } \\
\text { Wilson-Cook, } \\
\text { Winston-Salem, NC, USA) } \\
\text { Uncovered: Wallstent } \\
\text { (Schneider AG, Bülach, } \\
\text { Switzerland) or } \\
\text { Strecker-Stent (Boston } \\
\text { Scientific, Watertown, MA, } \\
\text { USA) }\end{array}$ \\
\hline Prat & 1998 & France & 1993-1995 & $\begin{array}{c}\text { Plastic: } 33 \\
\text { Uncovered: } 34\end{array}$ & $\begin{array}{c}\text { Plastic: mean } \\
73.3 \text { (range, } \\
\text { 39-92) } \\
\text { Uncovered: } \\
\text { mean 71.9 } \\
\text { (range, 39-95) }\end{array}$ & $\begin{array}{c}\text { Plastic: } 51.5 \\
\text { Uncovered: } 44.1\end{array}$ & N/A & $\begin{array}{c}\text { Plastic: pancreatic cancer } \\
(66.7 \%), \text { bile duct cancer } \\
(21.2 \%), \text { ampullary cancer } \\
(3.0 \%), \text { metastatic cancer } \\
(9.1 \%) \\
\text { Uncovered: pancreatic } \\
\text { cancer }(73.5 \%) \text {, bile duct } \\
\text { cancer }(14.7 \%), \text { ampullary } \\
\text { cancer }(0.0 \%) \text {, metastatic } \\
\text { cancer }(11.8 \%)\end{array}$ & $\begin{array}{c}\text { Plastic: mean } \\
13.2 \\
\text { Uncovered: } \\
\text { mean } 14.7\end{array}$ & $\begin{array}{c}\text { Plastic: } 11.5-\mathrm{Fr} \\
\text { polyethylene stent (Wilson } \\
\text { Cook, Winston-Salem, NC, } \\
\text { USA) } \\
\text { Uncovered: } 30-\mathrm{Fr} \\
\text { self-expanding metallic } \\
\text { stent (Wallsent, } \\
\text { Schneider-Howmedica, } \\
\text { Lyons, France) }\end{array}$ \\
\hline Kaassis & 2003 & France & 1997-1999 & $\begin{array}{c}\text { Plastic: } 59 \\
\text { Uncovered: } 59\end{array}$ & $\begin{array}{c}\text { Plastic: median } \\
75.9 \text { (IQR, } \\
66.9-84.3 \text { ) } \\
\text { Uncovered: } \\
\text { median 78.5 } \\
\text { (IQR, 70.0-87.9) }\end{array}$ & $\begin{array}{c}\text { Plastic: } 54.2 \\
\text { Uncovered: } 35.6\end{array}$ & $\mathrm{~N} / \mathrm{A}$ & $\begin{array}{c}\text { Plastic: pancreatic cancer } \\
(72.9 \%), \text { bile duct cancer } \\
(13.6 \%) \text {, metastatic cancer } \\
(11.9 \%) \text {, others }(1.7 \%) \\
\text { Uncovered: pancreatic } \\
\text { cancer }(76.3 \%) \text {, bile duct } \\
\text { cancer }(16.9 \%) \text {, metastatic } \\
\text { cancer }(5.1 \%) \text {, others } \\
(1.7 \%)\end{array}$ & $\begin{array}{c}\text { Plastic: median } \\
\text { 16.2 (IQR, } \\
7.7-20.2) \\
\text { Uncovered: } \\
\text { median 13.7 } \\
\text { (IQR, 6.7-18.0) }\end{array}$ & $\begin{array}{c}\text { Plastic: 10-Fr } \\
\text { Tannenbaum-type stent } \\
\text { (Soehendra ST-2, Wilson } \\
\text { Cook, Clarenton, France) } \\
\text { Uncovered: 10-mm metal } \\
\text { stent (Wallstent, Boston } \\
\text { Scientific Corp., St. } \\
\text { Quentin en Yvelines, } \\
\text { France) }\end{array}$ \\
\hline
\end{tabular}


Table 1. Cont.

\begin{tabular}{|c|c|c|c|c|c|c|c|c|c|c|}
\hline $\begin{array}{l}\text { First } \\
\text { Author }\end{array}$ & $\begin{array}{c}\text { Publication } \\
\text { Year }\end{array}$ & Country & $\begin{array}{l}\text { Study } \\
\text { Period }\end{array}$ & $\begin{array}{l}\text { Number of } \\
\text { Patients }\end{array}$ & $\begin{array}{c}\text { Age, Year, } \\
\text { Mean } \pm \text { SD }\end{array}$ & Male, n (\%) & $\begin{array}{l}\text { Performance } \\
\text { Status }\end{array}$ & Cause of Obstruction & $\begin{array}{c}\text { Baseline total } \\
\text { Bilirubin, } \\
\text { mg/dL, Mean } \pm \\
\text { SD }\end{array}$ & Used Stent \\
\hline Isayama & 2004 & Japan & 1998-2001 & $\begin{array}{l}\text { Uncovered: } 55 \\
\text { Covered: } 57\end{array}$ & $\begin{array}{c}\text { Uncovered: } \\
\text { mean 70.4 } \\
\text { (range, } 40-89 \text { ) } \\
\text { Covered: mean } \\
70.5 \text { (range, } \\
48-88 \text { ) }\end{array}$ & $\begin{array}{c}\text { Uncovered: } 56.4 \\
\text { Covered: } 61.4\end{array}$ & $\mathrm{~N} / \mathrm{A}$ & $\begin{array}{c}\text { Uncovered: pancreatic } \\
\text { cancer }(58.2 \%) \text {, bile duct } \\
\text { cancer }(9.1 \%), \text { gallbladder } \\
\text { cancer }(10.9 \%) \text {, ampullary } \\
\text { cancer }(1.8 \%), \text { metastatic } \\
\text { cancer }(20.0 \%) \\
\text { Covered: pancreatic cancer } \\
(59.6 \%) \text {, bile duct cancer } \\
(10.5 \%) \text {, gallbladder cancer } \\
(5.3 \%) \text {, ampullary cancer } \\
(3.5 \%), \text { metastatic cancer } \\
(21.1 \%)\end{array}$ & $\begin{array}{c}\text { Uncovered: } 8.5 \\
\pm 6.3 \\
\text { Covered: } 10.5 \pm \\
7.2\end{array}$ & $\begin{array}{l}\text { Uncovered: Uncovered } \\
\text { Ultraflex Diamond Stent } \\
\text { (Microvasive; Boston } \\
\text { Scientific Corporation, } \\
\text { Natik, Massachusetts, } \\
\text { USA) } \\
\text { Covered: Self-expandable } \\
\text { Ultraflex Diamond Stent } \\
\text { (Microvasive, Boston } \\
\text { Scientific Corporation, } \\
\text { Natik, Massachusetts, } \\
\text { USA) }\end{array}$ \\
\hline Katsinelos & 2006 & Greece & 2000-2005 & $\begin{array}{c}\text { Plastic: } 24 \\
\text { Uncovered: } 23\end{array}$ & $\begin{array}{c}\text { Plastic: median } \\
72 \text { (range, 56-82) } \\
\text { Uncovered: } \\
\text { median 74 } \\
\text { (range, 57-86) }\end{array}$ & $\begin{array}{c}\text { Plastic: } 41.7 \\
\text { Uncovered: } 60.9\end{array}$ & $\begin{array}{c}\text { ASA PS } \\
\text { classification } \\
\text { Plastic (II/III): } \\
45.8 \% / 54.2 \% \\
\text { Uncovered (II/III): } \\
39.1 \% / 60.9 \%\end{array}$ & $\begin{array}{c}\text { Plastic: pancreatic cancer } \\
(54.2 \%), \text { bile duct cancer } \\
(16.7 \%), \text { ampullary cancer } \\
(12.5 \%), \text { metastatic cancer } \\
(16.7 \%) \\
\text { Uncovered: pancreatic } \\
\text { cancer }(52.2 \%) \text {, bile duct } \\
\text { cancer }(17.4 \%) \text {, ampullary } \\
\text { cancer }(8.7 \%), \text { metastatic } \\
\text { cancer }(21.7 \%)\end{array}$ & $\begin{array}{l}\text { Plastic: median } \\
10.6 \text { (range, } \\
4.8-19.3 \text { ) } \\
\text { Uncovered: } \\
\text { median } 14.1 \\
\text { (range, 5.4-35.8) }\end{array}$ & $\begin{array}{c}\text { Plastic: Teflon } \\
\text { Tannenbaum stents } \\
\text { (Sohendra ST-2, Wilson } \\
\text { Cook, Winston-Salem, NC, } \\
\text { USA) } \\
\text { Uncovered: uncovered } \\
\text { self-expanding metal stent } \\
\text { (M.I. Tech, G.C. Medical } \\
\text { Co. Ltd., Pyungtaek, } \\
\text { Korea) }\end{array}$ \\
\hline Soderlund & 2006 & Sweden & $2002-2004$ & $\begin{array}{l}\text { Plastic: } 51 \\
\text { Covered: } 49\end{array}$ & $\begin{array}{c}\text { Plastic: median } \\
78 \text { (range, } 49-93 \text { ) } \\
\text { Covered: } \\
\text { median } 77 \\
\text { (range, } 48-92 \text { ) }\end{array}$ & $\begin{array}{c}\text { Plastic: } 54.9 \\
\text { Covered: } 44.9\end{array}$ & $\begin{array}{c}\text { ECOG } \\
\text { performance } \\
\text { status } \\
\text { Plastic } \\
(0 / 1 / 2 / 3 / 4): \\
15.7 \% / 37.3 \% / \\
31.4 \% / 11.8 \% / 3.9 \% \\
\text { Covered } \\
(0 / 1 / 2 / 3 / 4): \\
16.3 \% / 40.8 \% / \\
28.6 \% / 10.2 \% / 4.1 \%\end{array}$ & $\begin{array}{c}\text { Plastic: pancreatic cancer } \\
(74.5 \%) \text {, bile duct cancer } \\
(7.8 \%) \text {, ampullary cancer } \\
(2.0 \%) \text {, metastatic cancer } \\
(9.8 \%) \text {, others }(5.9 \%) \\
\text { Covered: pancreatic cancer } \\
(81.6 \%) \text {, bile duct cancer } \\
(10.2 \%) \text {, ampullary cancer } \\
(2.0 \%) \text {, metastatic cancer } \\
(4.1 \%) \text {, others }(2.0 \%)\end{array}$ & $\begin{array}{l}\text { Plastic: median } \\
13.9 \text { (range, } \\
0.5-36.8 \text { ) } \\
\text { Covered: } \\
\text { median } 11.6 \\
\text { (range, } 1.1-40.1 \text { ) }\end{array}$ & $\begin{array}{l}\text { Plastic: 10-Fr polyethylene } \\
\text { endoprothesis (Boston } \\
\text { Scientific Nordic AB, } \\
\text { Helsingborg, Sweden) } \\
\text { Covered: Wallstent } \\
\text { (Boston Scientific Nordic } \\
\text { AB, Helsingborg, Sweden) }\end{array}$ \\
\hline
\end{tabular}


Table 1. Cont.

\begin{tabular}{|c|c|c|c|c|c|c|c|c|c|c|}
\hline $\begin{array}{l}\text { First } \\
\text { Author }\end{array}$ & $\begin{array}{l}\text { Publication } \\
\text { Year }\end{array}$ & Country & $\begin{array}{l}\text { Study } \\
\text { Period }\end{array}$ & $\begin{array}{l}\text { Number of } \\
\text { Patients }\end{array}$ & $\begin{array}{c}\text { Age, Year, } \\
\text { Mean } \pm \text { SD }\end{array}$ & Male, n (\%) & $\begin{array}{l}\text { Performance } \\
\text { Status }\end{array}$ & Cause of Obstruction & $\begin{array}{l}\text { Baseline total } \\
\text { Bilirubin, } \\
\text { mg/dL, Mean } \pm \\
\text { SD }\end{array}$ & Used Stent \\
\hline Krokidis & 2010 & $\begin{array}{l}\text { Italy and } \\
\text { Greece }\end{array}$ & 2005-2007 & $\begin{array}{c}\text { Uncovered: } 30 \\
\text { Covered: } 30\end{array}$ & $\begin{array}{c}\text { Uncovered: } \\
\text { median 63.7 } \\
\text { (range, 46-73) } \\
\text { Covered: } \\
\text { median 66.5 } \\
\text { (range, 52-78) }\end{array}$ & $\begin{array}{c}\text { Uncovered: } 53.3 \\
\text { Covered: } 66.7\end{array}$ & N/A & $\begin{array}{c}\text { Uncovered: bile duct } \\
\text { cancer }(100.0 \%) \\
\text { Covered: bile duct cancer } \\
(100.0 \%)\end{array}$ & $\begin{array}{l}\text { Uncovered: } 7.2 \\
\text { Covered: } 10.3\end{array}$ & $\begin{array}{l}\text { Uncovered: 10-mm } \\
\text { Uncoverd Wallstent } \\
\text { Covered: 8- to 10-mm } \\
\text { Viabil Biliary Stent }\end{array}$ \\
\hline Telford & 2010 & USA & $2002-2008$ & $\begin{array}{c}\text { Uncovered: } 61 \\
\text { Covered: } 68\end{array}$ & $\begin{array}{l}\text { Uncovered: } 65 \\
\quad \pm 13 \\
\text { Covered: } 66 \pm \\
14\end{array}$ & $\begin{array}{c}\text { Uncovered: } 50.8 \\
\text { Covered: } 44.1\end{array}$ & $\begin{array}{c}\text { Karnofsky } \\
\text { performance score } \\
\text { Uncovered: mean } \\
74 \text { (SD, 17) } \\
\text { Covered: mean } 77 \\
\text { (SD, 18) }\end{array}$ & $\begin{array}{c}\text { Uncovered: pancreatic } \\
\text { cancer }(77.0 \%) \text {, others } \\
(33.0 \%) \\
\text { Covered: pancreatic cancer } \\
(86.8 \%) \text {, others }(13.2 \%)\end{array}$ & N/A & $\begin{array}{c}\text { Uncovered: uncovered } \\
\text { Wallstent (Boston Scientific } \\
\text { Corporation, Natick, MA, } \\
\text { USA) } \\
\text { Covered: permalume } \\
\text { partially covered Wallstent } \\
\text { (Boston Scientific } \\
\text { Corporation, Natick, MA, } \\
\text { USA) }\end{array}$ \\
\hline
\end{tabular}


Table 1. Cont.

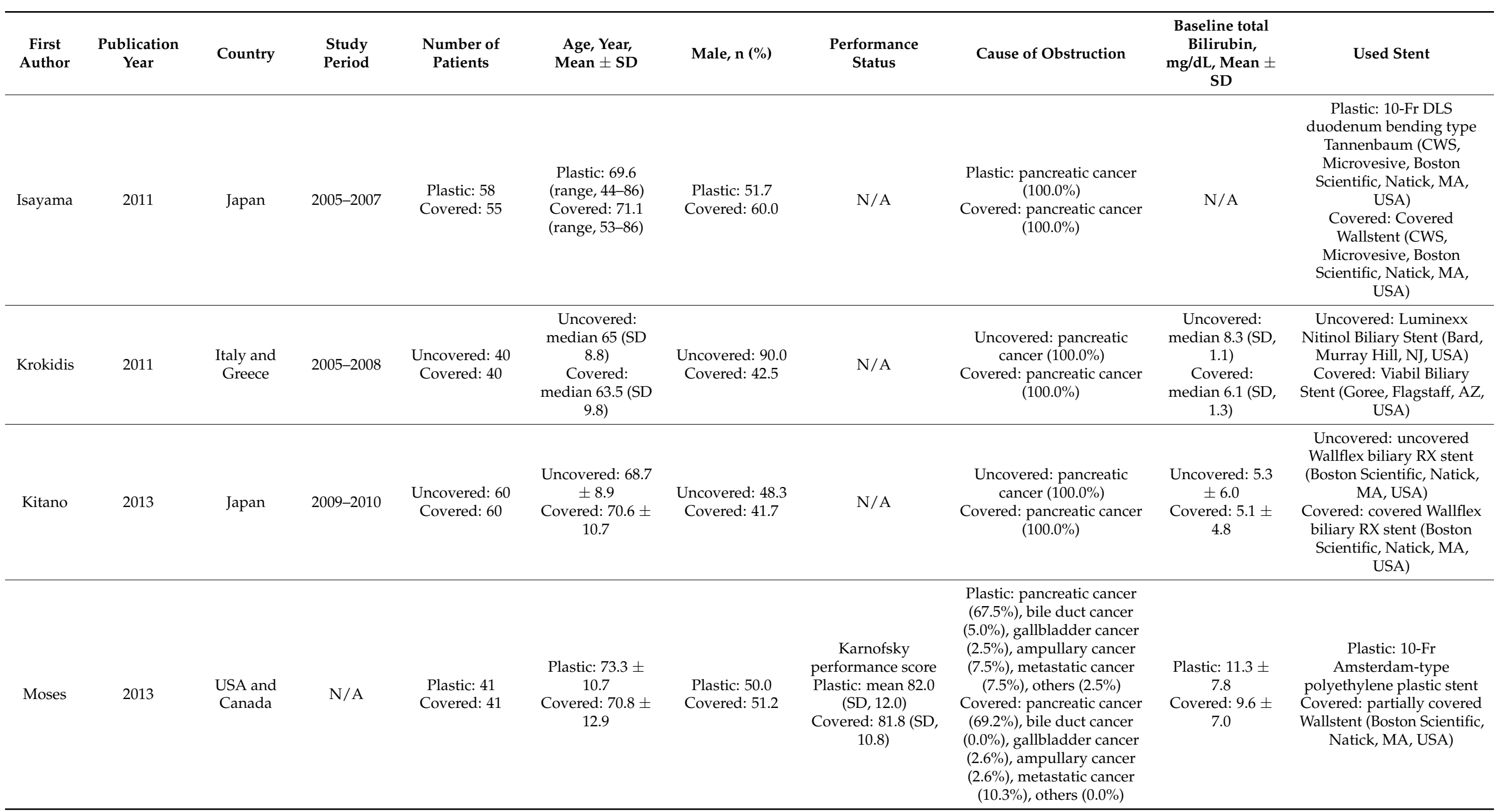


Table 1. Cont.

\begin{tabular}{|c|c|c|c|c|c|c|c|c|c|c|}
\hline $\begin{array}{l}\text { First } \\
\text { Author }\end{array}$ & $\begin{array}{c}\text { Publication } \\
\text { Year }\end{array}$ & Country & $\begin{array}{l}\text { Study } \\
\text { Period }\end{array}$ & $\begin{array}{l}\text { Number of } \\
\text { Patients }\end{array}$ & $\begin{array}{c}\text { Age, Year, } \\
\text { Mean } \pm \text { SD }\end{array}$ & Male, n (\%) & $\begin{array}{c}\text { Performance } \\
\text { Status }\end{array}$ & Cause of Obstruction & $\begin{array}{c}\text { Baseline total } \\
\text { Bilirubin, } \\
\text { mg/dL, Mean } \pm \\
\text { SD }\end{array}$ & Used Stent \\
\hline Ung & 2013 & Sweden & 2006-2009 & $\begin{array}{l}\text { Uncovered: } 34 \\
\text { Covered: } 34\end{array}$ & $\begin{array}{c}\text { Uncovered: } \\
\text { median } 79 \text { (IQR, } \\
64-83) \\
\text { Covered: } \\
\text { median 77 (IQR, } \\
67-83)\end{array}$ & $\begin{array}{c}\text { Uncovered: } 26.5 \\
\text { Covered: } 52.9\end{array}$ & N/A & $\begin{array}{c}\text { Uncovered: pancreatic } \\
\text { cancer }(79.4 \%) \text {, gallbladder } \\
\text { cancer }(14.7 \%), \text { ampullary } \\
\text { cancer }(8.8 \%) \text {, others }(2.9 \%) \\
\text { Covered: pancreatic } \\
\text { cancer: }(88.2 \%), \\
\text { gallbladder cancer }(5.9 \%), \\
\text { ampullary cancer }(2.9 \%), \\
\text { others }(5.9 \%)\end{array}$ & $\begin{array}{c}\text { Uncovered: } \\
\text { median } 10.6 \\
\text { (IQR, 1.7-14.2) } \\
\text { Covered: } \\
\text { median 4.6 (IQR, } \\
\text { 1.8-15.2) }\end{array}$ & $\begin{array}{l}\text { Uncovered: uncovered } \\
\text { Hanarostent (MI-tech, } \\
\text { Seoul, Korea) } \\
\text { Covered: covered } \\
\text { Hanarostent (MI-tech, } \\
\text { Seoul, Korea) }\end{array}$ \\
\hline Lee & 2014 & Korea & 2012-2013 & $\begin{array}{l}\text { Uncovered: } 20 \\
\text { Covered: } 20\end{array}$ & $\begin{array}{c}\text { Uncovered: } 63.2 \\
\pm 11.7 \\
\text { Covered: } 62.1 \pm \\
8.6\end{array}$ & $\begin{array}{c}\text { Uncovered: } 45.0 \\
\text { Covered: } 45.0\end{array}$ & N/A & $\begin{array}{c}\text { Uncovered: pancreatic } \\
\text { cancer }(30.0 \%) \text {, bile duct } \\
\text { cancer }(5.0 \%), \text { gallbladder } \\
\text { cancer }(15.0 \%) \text {, others } \\
(50.0 \%) \\
\text { Covered: pancreatic cancer } \\
(60.0 \%), \text { bile duct cancer } \\
(5.0 \%) \text {, gallbladder cancer } \\
(0.0 \%) \text {, others }(35.0 \%)\end{array}$ & $\begin{array}{c}\text { Uncovered: } 8.0 \\
\pm 6.6 \\
\text { Covered: } 6.2 \pm \\
4.9\end{array}$ & $\begin{array}{c}\text { Uncovered: Zilver } \\
\text { self-expanding stent (Cook, } \\
\text { Bloomington, IN, USA) } \\
\text { Covered: Niti-S stent, } \\
\text { ComVi type (Taewoong } \\
\text { Medical Co., Ltd., Seoul, } \\
\text { Korea) }\end{array}$ \\
\hline Walter & 2015 & Netherlands & 2008-2013 & $\begin{array}{l}\text { Plastic: } 57 \\
\text { Uncovered: } 60 \\
\text { Covered: } 54\end{array}$ & N/A & N/A & N/A & $\mathrm{N} / \mathrm{A}$ & N/A & $\begin{array}{c}\text { Plastic: } 10-F r \text { polyurethane } \\
\text { or polyethylene stent } \\
\text { (Boston Scientific } \\
\text { Corporation, Natick, MA, } \\
\text { USA) } \\
\text { Uncovered: } 10-m m \\
\text { uncovered Wallstent RX } \\
\text { (Boston Scientific } \\
\text { Corporation, Natick, MA, } \\
\text { USA) } \\
\text { Covered: 10-mm covered } \\
\text { Wallstent RX (Boston } \\
\text { Scientific Corporation, } \\
\text { Natick, MA, USA) }\end{array}$ \\
\hline
\end{tabular}


Table 1. Cont.

\begin{tabular}{|c|c|c|c|c|c|c|c|c|c|c|}
\hline $\begin{array}{l}\text { First } \\
\text { Author }\end{array}$ & $\begin{array}{c}\text { Publication } \\
\text { Year }\end{array}$ & Country & $\begin{array}{l}\text { Study } \\
\text { Period }\end{array}$ & $\begin{array}{l}\text { Number of } \\
\text { Patients }\end{array}$ & $\begin{array}{c}\text { Age, Year, } \\
\text { Mean } \pm \text { SD }\end{array}$ & Male, n (\%) & $\begin{array}{l}\text { Performance } \\
\text { Status }\end{array}$ & Cause of Obstruction & $\begin{array}{c}\text { Baseline total } \\
\text { Bilirubin, } \\
\text { mg/dL, Mean } \pm \\
\text { SD }\end{array}$ & Used Stent \\
\hline Yang & 2015 & Korea & 2006-2013 & $\begin{array}{c}\text { Uncovered: } 52 \\
\text { Covered: } 51\end{array}$ & $\begin{array}{c}\text { Uncovered: } 68.0 \\
\pm 11.3 \\
\text { Covered: } 68.7 \pm \\
11.2\end{array}$ & $\begin{array}{c}\text { Uncovered: } 57.7 \\
\text { Covered: } 66.7\end{array}$ & $\begin{array}{c}\text { Karnofsky } \\
\text { performance score } \\
\text { Uncovered: mean } \\
79.4 \text { (SD, 7.3) } \\
\text { Covered: mean } \\
78.0(\text { SD, } 9.0)\end{array}$ & $\begin{array}{c}\text { Uncovered: pancreatic } \\
\text { cancer }(69.2 \%) \text {, bile duct } \\
\text { cancer }(13.5 \%) \text {, gallbladder } \\
\text { cancer }(9.6 \%) \text {, ampullary } \\
\text { cancer }(3.8 \%) \text {, others }(3.8 \%) \\
\text { Covered: pancreatic cancer } \\
\text { (56.9\%), bile duct cancer } \\
(33.3 \%) \text {, gallbladder cancer } \\
(3.9 \%) \text {, ampullary cancer } \\
(3.9 \%) \text {, others }(2.0 \%)\end{array}$ & $\begin{array}{c}\text { Uncovered: } 11.4 \\
\pm 6.1 \\
\text { Covered: } 10.7 \pm \\
8.4\end{array}$ & $\begin{array}{l}\text { Uncovered: Uncovered } \\
\text { Bonastent (Standard } \\
\text { Sci-Tech Inc., Seoul, Korea) } \\
\text { Covered: partially covered } \\
\text { Bonastent (Standard } \\
\text { Sci-Tech Inc., Seoul, Korea) }\end{array}$ \\
\hline Bernon & 2018 & South Africa & 2009-2013 & $\begin{array}{c}\text { Plastic: } 19 \\
\text { Uncovered: } 21\end{array}$ & $\begin{array}{c}\text { Plastic: median } \\
65 \text { (IQR, 60-80) } \\
\text { Uncovered: } \\
\text { median 69.5 } \\
\text { (IQR, 59.5-74) }\end{array}$ & $\begin{array}{c}\text { Plastic: } 42.1 \\
\text { Uncovered: } 42.9\end{array}$ & $\begin{array}{c}\text { ECOG } \\
\text { performance } \\
\text { status } \\
\text { Plastic }(0 / 1 / 2): \\
0.0 \% / 21.1 \% / 78.9 \% \\
\text { Uncovered } \\
\text { (0/1/2): } \\
4.8 \% / 23.8 \% / 71.4 \%\end{array}$ & $\begin{array}{c}\text { Plastic: pancreatic cancer } \\
(89.5 \%), \text { bile duct cancer } \\
(10.5 \%) \\
\text { Uncovered: pancreatic } \\
\text { cancer }(85.7 \%), \text { bile duct } \\
\text { cancer }(14.3 \%)\end{array}$ & $\begin{array}{c}\text { Plastic: median } \\
\text { 19.8 (range, } \\
4.2-38.1 \text { ) } \\
\text { Uncovered: } \\
\text { median 20.9 } \\
\text { (range, } 2.3-39.8 \text { ) }\end{array}$ & $\begin{array}{c}\text { Plastic: standard } \\
\text { polyethylene plastic stent } \\
\text { (Boston Scientific, Natick, } \\
\text { MA, USA) } \\
\text { Uncovered: 10-mm } \\
\text { uncovered stent (Boston } \\
\text { Scientific, Natick, MA, } \\
\text { USA) }\end{array}$ \\
\hline Dhondt & 2020 & Belgium & 2002-2018 & $\begin{array}{c}\text { Uncovered: } 78 \\
\text { Covered: } 73\end{array}$ & $\begin{array}{l}\text { Uncovered: } 69 \\
\text { (range, 22-88) } \\
\text { Covered: 68 } \\
\text { (range, 35-90) }\end{array}$ & $\begin{array}{l}\text { Uncovered: } 43.6 \\
\text { Covered: } 41.1\end{array}$ & N/A & $\begin{array}{c}\text { Uncovered: pancreatic } \\
\text { cancer }(57.7 \%) \text {, bile duct } \\
\text { cancer }(2.6 \%) \text {, duodenal } \\
\text { cancer }(1.3 \%) \text {, metastatic } \\
\text { cancer (38.5\%) } \\
\text { Covered: pancreatic cancer } \\
\text { (61.6\%), bile duct cancer } \\
(0.0 \%) \text {, duodenal cancer } \\
(0.0 \%) \text {, metastatic cancer } \\
(38.4 \%)\end{array}$ & $\begin{array}{c}\text { Uncovered: } 13.0 \\
\pm 0.9 \\
\text { Covered: } 13.0 \pm \\
0.8\end{array}$ & $\begin{array}{c}\text { Uncovered: ZA biliary } \\
\text { stent (Cook Europe, } \\
\text { Limerick, Ireland) } \\
\text { Covered: VIABIL } \\
\text { endoprosthesis (W.L. Gore } \\
\text { \& Associates, Flagstaff, } \\
\text { Arizona, USA) }\end{array}$ \\
\hline
\end{tabular}

ECOG, Eastern Cooperative Oncology Group; ASA PS, American Society of Anesthesiologist physical status; SD, standard deviation; IQR, interquartile range; CI, confidence interval; N/A, not available. 
The evidence network is shown in Figure S1.

Lines represent the comparison between stent types. The thickness of these lines and the numbers shown in large represent the number of studies included in each comparison. The numbers in rectangles indicate the number of patients included in each comparison. Because one study was a three-arm trial, the sum of comparisons and that of patients exceeded the number of included studies and that of included patients, respectively. SEMS, self-expandable metal stent.

The risk of bias assessments for individual studies is shown in Figure S2.

Among the 21 included studies, $3(14.3 \%)$ had an unclear risk of bias regarding random sequence generation. An unclear risk of bias in terms of allocation concealment was identified in 8 studies (38.1\%). All studies were assessed as having a low risk of performance and detection bias because stent-related outcomes, including RBO, are less likely to be affected by the blinding of participants and investigators. Attrition bias was not identified. One study (4.8\%) was assessed as having a high risk of reporting bias because it did not report the proportion of $\mathrm{RBO}$, which was a primary endpoint of our meta-analysis.

\subsection{Technical and Clinical Success According to the Stent Type}

Technical and clinical successes were excellent regardless of stent type. The proportion of technical success was 99.4\% (95\% CI, 98.6-99.7\%) in the plastic stent group, 100.0\% (95\% CI, 98.5-100.0\%) in the uncovered SEMS group, and 99.2\% (95\% CI, 98.6-99.7\%) in the covered SEMS group. Additionally, the proportion of clinical success was $90.1 \%$ (95\% CI, 84.9-93.6\%) in the plastic stent group, 94.6\% (95\% CI, 91.2-96.7\%) in the uncovered SEMS group, and $92.3 \%$ (95\% CI, 88.4-94.9\%) in the covered SEMS group. There was no statistical difference in technical and clinical successes among stent types.

\subsection{Direct Meta-Analysis for Recurrent Biliary Obstruction and Stent-Related Inflammation}

Figure 2 demonstrates the comparative efficacy in terms of RBO between any two stent types. Both the uncovered and covered SEMS groups showed a lower risk of RBO compared to the plastic stent group without statistically significant heterogeneity (uncovered SEMS vs. plastic stent: $\mathrm{RR}(95 \% \mathrm{CI})=0.44(0.33-0.57), \mathrm{df}=6, p=0.35, I^{2}=11 \%$; and covered SEMS vs. plastic stent: RR $\left.(95 \% \mathrm{CI})=0.51(0.38-0.68), \mathrm{df}=3, p=0.39, I^{2}=0 \%\right)$.

Between the uncovered and covered SEMS groups, there was no significant difference in terms of RBO, but there was statistical heterogeneity (covered SEMS vs. uncovered SEMS: RR $\left.(95 \% \mathrm{CI})=0.93(0.68-1.26), \mathrm{df}=10, p=0.009, I^{2}=57 \%\right)$. Publication bias was assessed for RBO between the uncovered and covered SEMS groups. We did not detect any corresponding publication bias using the Begg and Mazumdar-adjusted rank correlation tests $(p=0.938)$. Asymmetry was not observed upon the visual inspection of the funnel plot (Figure S3).

The white diamond represents the pooled logarithmic RR, with a 95\% confidence interval, among observed studies.

RBO, recurrent biliary obstruction; SEMS, self-expandable metal stent; M-H, MantelHaenszel; RR, risk ratio

Figure S4 shows forest plots for causes of RBO, including stent occlusion by sludge, tumor ingrowth, tumor overgrowth, and stent migration. Stent occlusion by sludge was common in the plastic stent group, tumor ingrowth was common in the uncovered SEMS group, and tumor overgrowth and stent migration tended to be common in the covered SEMS group. M-H, Mantel-Haenszel; CI, confidence interval.

In terms of stent-related inflammation, there was no statistical difference in most comparisons (Figure S5). Although cholangitis was less common in the covered SEMS group than in the plastic stent group, only one study was included in that comparison. $\mathrm{M}-\mathrm{H}$, Mantel-Haenszel; CI, confidence interval. 


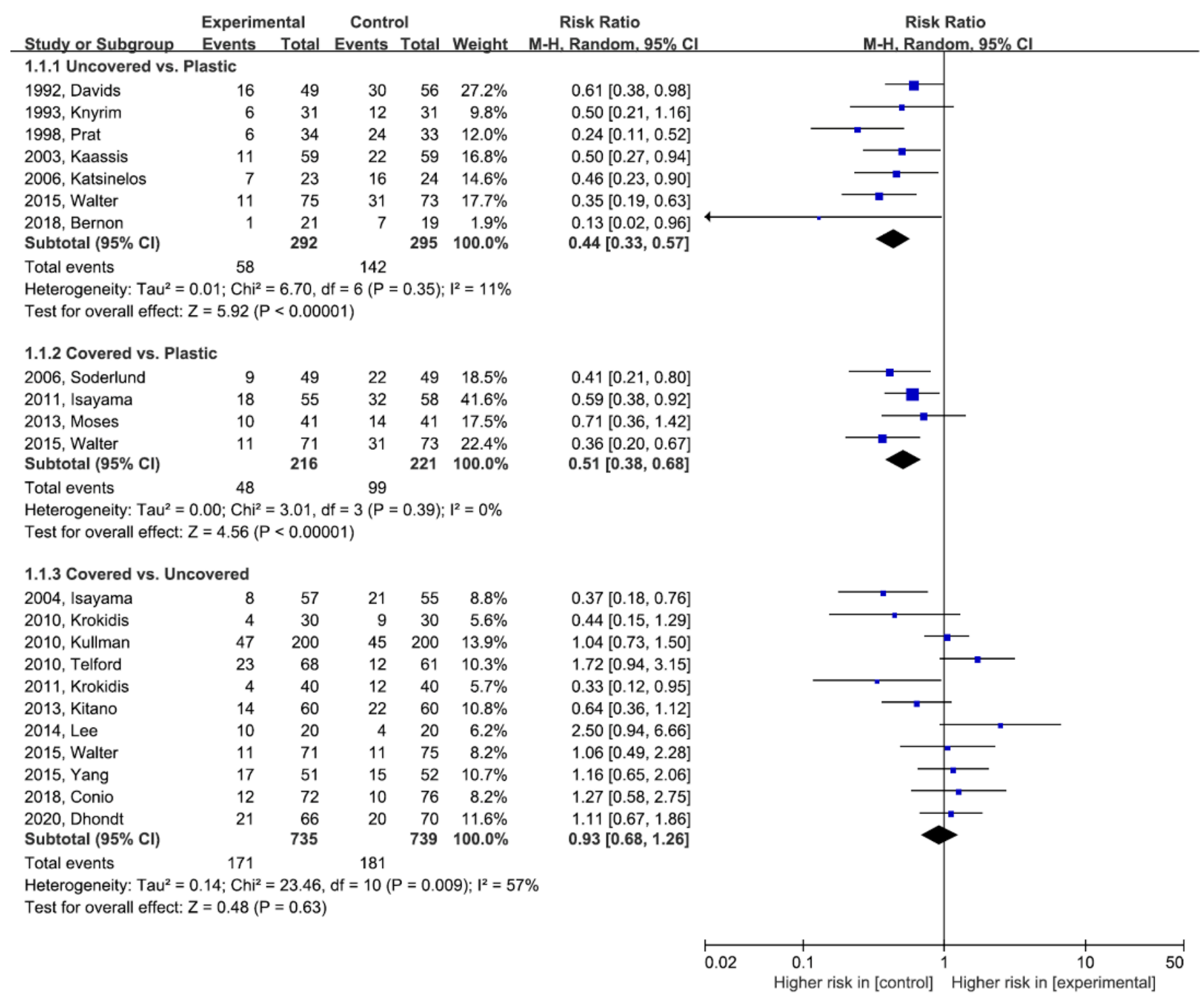

Figure 2. Direct meta-analysis for recurrent biliary obstruction. M-H, Mantel-Haenszel; CI, confidence interval.

\subsection{Network Meta-Analysis for Recurrent Biliary Obstruction and Stent-Related Inflammation}

In Figure 3, forest plots show the network estimates of RBO and its causes in the uncovered and covered SEMS groups compared with the plastic stent group. Both uncovered and covered SEMS groups showed a lower risk of RBO compared to the plastic stent group (RR (95\% CI): uncovered SEMS vs. plastic stent, 0.46 (0.35-0.62); and covered SEMS vs. plastic stent, $0.46(0.34-0.62))$. There was no significant difference between the uncovered and covered SEMS groups (uncovered vs. covered: RR $(95 \% \mathrm{CI})=1.02(0.80-1.30)$ ). Plastic stents had the highest P-score ( $>99 \%)$, followed by uncovered SEMS $(28 \%)$ and covered SEMS (22\%).

The detailed efficacy profiles of stents are summarized in Table S1. There was no network inconsistency in the comparison.

Both uncovered and covered SEMS groups also had a lower risk of stent occlusion by sludge compared to the plastic stent group (RR ( $95 \% \mathrm{CI}$ ): uncovered SEMS vs. plastic stent, 0.09 (0.04-0.18); and covered SEMS vs. plastic stent, 0.17 (0.08-0.37)). Additionally, the risk of stent occlusion by sludge in the uncovered SEMS group was lower than that in the covered SEMS group (uncovered vs. covered: RR $(95 \% \mathrm{CI})=0.51(0.31-0.83)$ ). However, the uncovered SEMS group had a higher risk of tumor ingrowth compared to the covered SEMS and plastic stent groups (RR $(95 \% \mathrm{CI})$ : uncovered SEMS vs. covered SEMS, 4.49 
(2.21-9.09); and uncovered SEMS vs. plastic stent, 23.79 (2.54-222.81)). In terms of tumor overgrowth, both uncovered and covered SEMS groups tended to be higher risk compared to the plastic stent group. However, the covered SEMS group had a significantly higher risk of tumor overgrowth compared to the uncovered SEMS group (covered vs. uncovered: RR $(95 \% \mathrm{CI})=1.98(1.18-3.34))$. Stent migration was common in the covered SEMS group than in the uncovered SEMS group (covered vs. uncovered: RR $(95 \% \mathrm{CI})=8.38(2.83-24.84)$ ). There was no network inconsistency in any comparison.

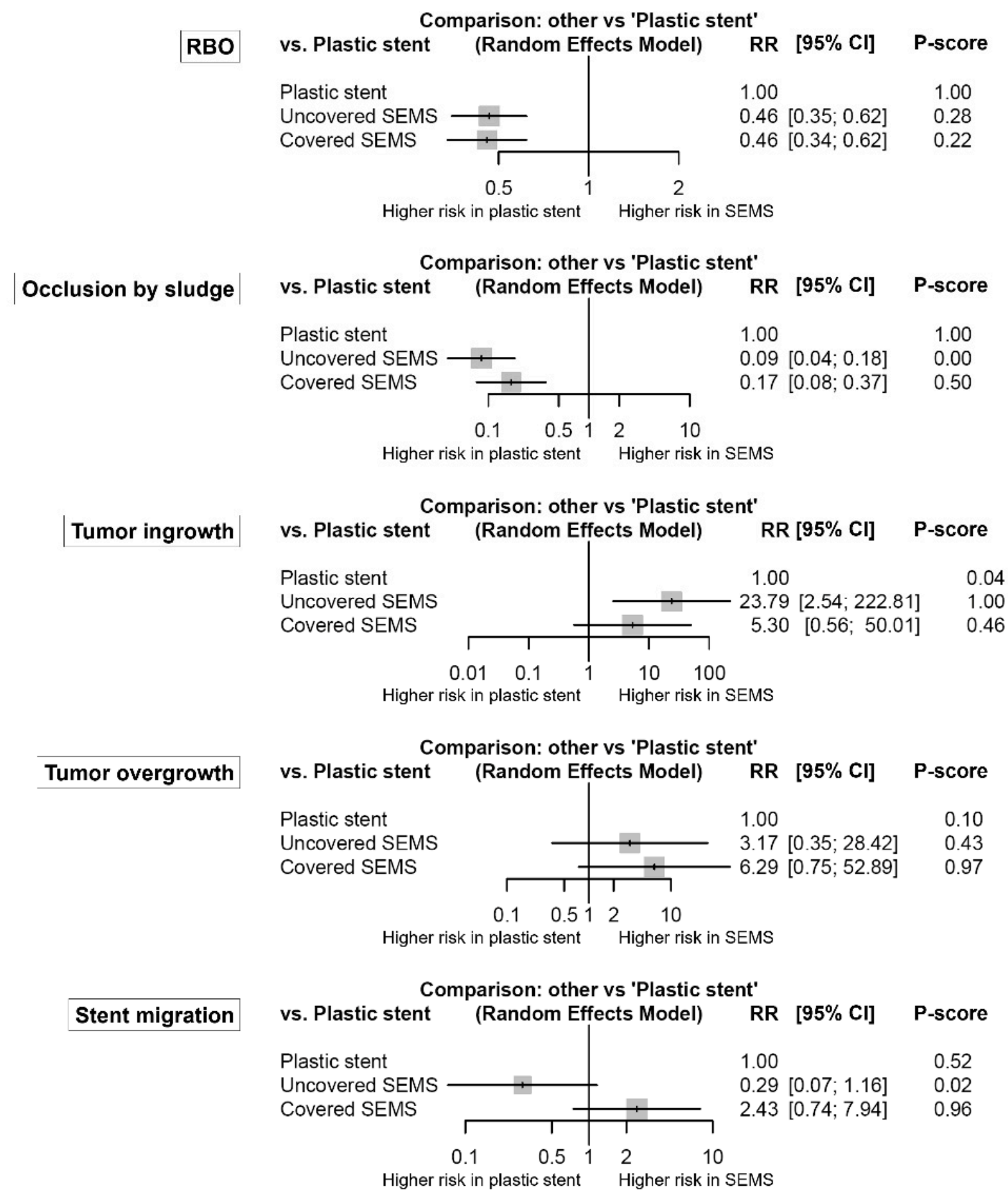

Figure 3. Comparative efficacy for recurrent biliary obstruction and its causes according to the type of stents from the network meta-analysis. The P-score indicates the mean extent of certainty that one type of stent is better than another. RBO, recurrent biliary obstruction; SEMS, self-expandable metal stent; RR, risk ratio; CI, confidence interval. 
Figure 4 shows the forest plots for stent-related inflammation. Although cholangitis was marginally more common in the plastic stent group than in the covered SEMS group $(\mathrm{RR}(95 \% \mathrm{CI})=2.37(1.003-5.60))$, there was no significant difference or network inconsistency in any other comparison.

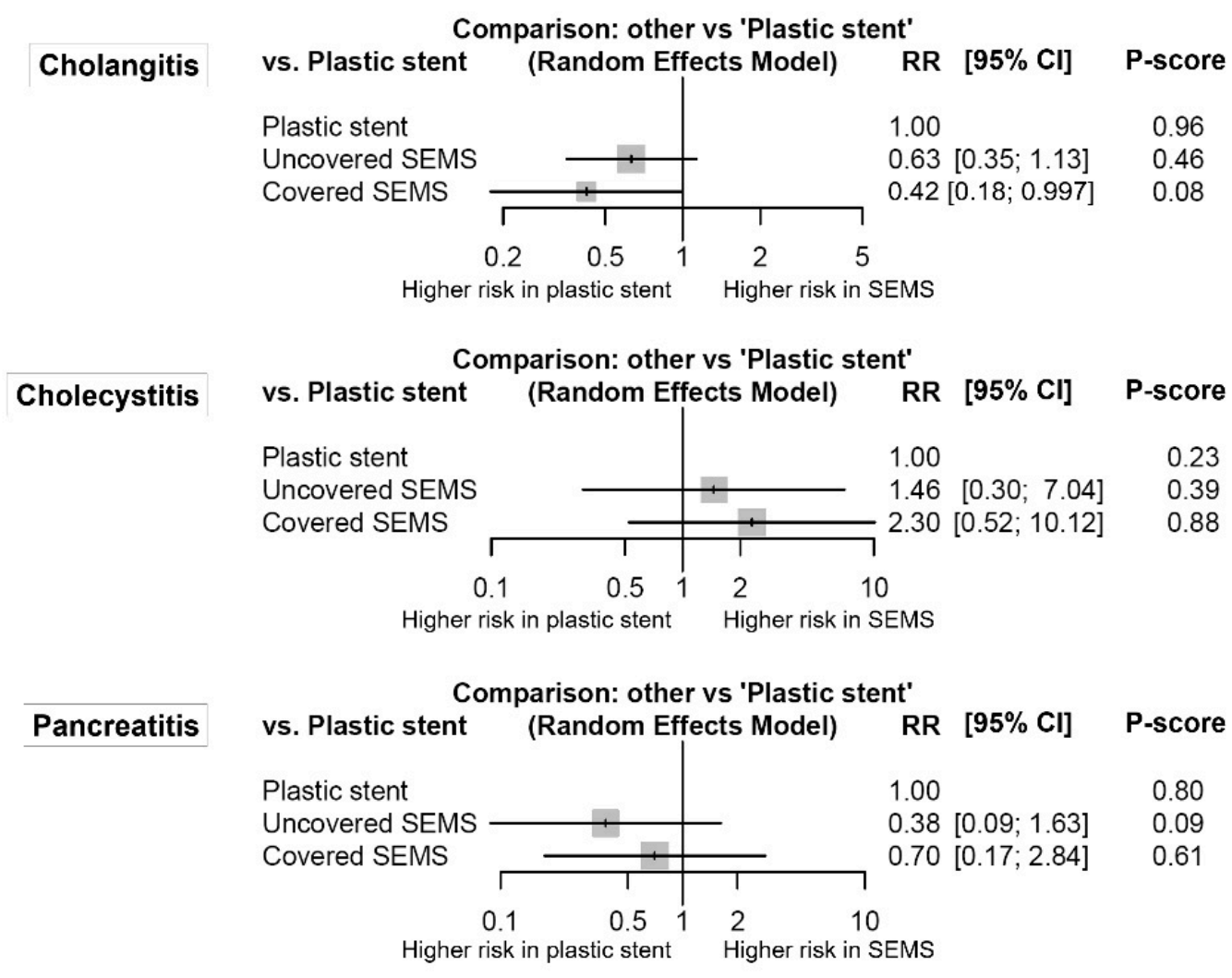

Figure 4. Comparative efficacy for stent-related inflammation according to the type of stents from the network meta-analysis. The P-score indicates the mean extent of certainty that one type of stent is better than another. SEMS, self-expandable metal stent; $\mathrm{RR}$, risk ratio; $\mathrm{CI}$, confidence interval.

\subsection{Absolute Risk of Recurrent Biliary Obstruction and Adverse Events}

To understand the absolute risk of RBO and adverse events, the incidence of each outcome was summarized in Table S2. The proportion of RBO was $47.4 \%$ (95\% CI, $42.8-$ $52.1 \%)$ in the plastic stent group, $23.8 \%(95 \% \mathrm{CI}, 21.3-26.7 \%)$ in the uncovered SEMS group, and $23.6 \%(95 \% \mathrm{CI}, 20.9-26.6 \%)$ in the covered SEMS group. RBO was mainly caused by sludge in the plastic stent group (incidence, $44.8 \%$ (95\% CI, 37.3-52.5\%)) and tumor ingrowth in the uncovered SEMS group (incidence, 17.3\% (95\% CI, 14.5-20.4\%)). In the covered SEMS group, stent occlusion by sludge, tumor overgrowth, and stent migration was found in $7.5 \%, 7.1 \%$, and $5.0 \%$ of patients, respectively. The proportion of cholangitis was $36.3 \%$ (95\% CI, 29.4-43.7\%) in the plastic stent group, $12.1 \%$ (95\% CI, 9.5-15.4\%) in the uncovered SEMS group, and 5.2\% (95\% CI, 3.4-7.9\%) in the covered SEMS group.

\section{Discussion}

This network meta-analysis study is the first to comprehensively compare three types of stents for the endoscopic drainage of extrahepatic MBO. Compared to the traditional pairwise meta-analytic approach, our study has the innovative advantage of filling the literature gap between RCTs, offering the application of appropriate stents to the clinical field through novel comparisons. We showed that all three types of stents have extremely high technical success rates of more than $99 \%$. Additionally, all stent types showed high clinical success rates of more than $90 \%$. However, the clinical success rate of the plastic stent tended to be $4.5 \%$ lower than that of the uncovered SEMS $(90.1 \%$ vs. $94.6 \%)$. This 
might be derived from the vulnerability of the plastic stent and sludge occlusion, followed by biliary obstruction, subsequent cholangitis, and the need for stent revision [40,41].

Regarding primary outcomes related to RBO, both uncovered and covered SEMSs showed a lower risk compared to plastic stents. The diameter of the stent may play an essential role in maintaining stent patency; thus, a 10-Fr plastic stent would be better than 7- or 8-Fr stents [42]. However, duodenoscopes have a limitation of delivery for plastic stents greater than about 11.5-Fr stents through the working channel. In contrast, biliary SEMSs with larger internal diameters (up to $10 \mathrm{~mm}$ ) can be placed with a 7- or 8-Fr delivery system, which is enough to pass through the working channel [43].

RBO did not differ between uncovered and covered SEMSs. However, it was affected by different mechanisms. Covered SEMSs were more likely to result in RBO from sludge occlusion, tumor overgrowth, and stent migration, whereas uncovered SEMSs were mainly influenced by tumor ingrowth. The coating membrane of the covered SEMS was invented to prevent tumor ingrowth; however, it offers a medium for the attachment and colonization of a bacterial biofilm [9], which lead to stent sludge occlusion. Simultaneously, it can disturb the embedding and anchoring of the stent in situ [44]. This can consequently lead to an 8.4-fold higher chance of stent migration compared with uncovered SEMSs. However, the absolute risk of stent migration was relatively low even in patients who underwent covered SEMS placement (5.0\%) compared with that of tumor ingrowth caused by uncovered SEMSs (17.3\%).

Although SEMSs have a relatively good stent patency, their revision for RBO is challenging compared to plastic stents because of difficulties in stent removal or replacement, especially when an uncovered SEMS was placed. Thus, plastic stents might be appropriate for patients with a life expectancy of less than six months, whereas SEMS can be more favorable for patients with a life expectancy of six months or more [45].

Among stent-related inflammations, including cholangitis, cholecystitis, and pancreatitis, cholangitis may be the most concerning because it is the most common. The risk of cholangitis in the plastic stent group was higher than that in the covered SEMS group. Because biliary drainage through plastic stents may require more re-interventions than drainage through SEMSs, the risk of cholangitis can be increased when plastic stents are placed. Regarding cholecystitis, several studies reported that covered SEMSs might have a higher cholecystitis risk after biliary stent placement than after uncovered SEMS placement $[46,47]$. Additionally, our meta-analysis showed a tendency of increased cholecystitis risk in the covered SEMS group compared to the plastic stent group; however, there was no significant difference between groups. It was difficult to identify the small differences regarding cholecystitis risk among stents because incidences of stent-related cholecystitis were very low ( $0.5 \%$ for plastic stents, $1.6 \%$ for uncovered SEMSs, and 3.1\% for covered SEMSs). Additionally, it has been suggested that tumor invasions to the feeding artery of the gallbladder or the orifice of the cystic duct, rather than the stent itself, might contribute to the development of cholecystitis [48]. Meanwhile, there is also a concern regarding acute pancreatitis surrounding SEMSs because the opening of the pancreatic duct could, theoretically, be occluded by the axial force of the stent $[49,50]$. However, our analysis showed no statistical difference in the risk of pancreatitis among stents.

Although this study was the first network meta-analysis to compare the efficacies of various stents in the palliative drainage for extrahepatic $\mathrm{MBO}$, it has several limitations. First, significant heterogeneity was identified in the comparisons of uncovered and covered SEMSs regarding RBO and tumor ingrowth; however, there was no significant heterogeneity in any other comparisons. Second, the specific models of the compared stents varied across the included studies. Although the inclusion of various stent models may help generalize our study findings, it may be a potential confounding variable. A multicenter-cohort study reported that covered WallFlex stents were superior to covered Wallstents in terms of the cumulative incidence of RBO [51]. A Korean multicenter study showed different migration risk between covered SEMS with flaps and conventional covered SEMS in patients with benign biliary strictures [52]. Third, biliary obstructions in 
the included patients were caused by various underlying malignant diseases, including pancreatic cancer, bile duct cancer, gallbladder cancer, and ampullary cancer. Because clinical outcomes and adverse events in each included study were reported without the consideration of underlying diseases, we could not perform a subgroup analysis according to the types of malignant diseases.

Despite these limitations, the present network meta-analysis provides a better understanding of clinical outcomes and adverse events according to the type of stents in patients with extrahepatic MBO. In conclusion, we recommend that the use of an SEM should be considered as the first option to treat extrahepatic MBOs because SEMs have longer stent patency, lower sludge occlusion rate, and lower risk of stent-related cholangitis, compared with plastic stents. Uncovered SEMSs had a high risk of tumor ingrowth, whereas covered SEMSs had a high risk of stent migration, tumor overgrowth, and sludge occlusion. However, the overall risk of RBO was similar between the uncovered and covered SEMSs.

Supplementary Materials: The following are available online at https:/ / www.mdpi.com/2075-4 426/11/2/86/s1, Figure S1: Evidence network of different stents. Lines represent the comparison between stent types. The thickness of these lines and the numbers shown in large represent the number of studies included in each comparison. The numbers in rectangles indicate the number of patients included in each comparison. Because one study was a three-arm trial, the sum of comparisons and that of patients exceeded the number of included studies and that of included patients, respectively. SEMS, self-expandable metal stent. Figure S2: Risk of bias summary: the review authors' judgments regarding the risk of bias items for each included study. Figure S3: Funnel plot for the analysis of recurrent biliary obstruction between the uncovered and covered SEMS groups. Figure S4: Direct meta-analysis for causes of recurrent biliary obstruction. (A) sludge, (B) tumor ingrowth, (C) tumor overgrowth, and (D) stent migration. Figure S5: Direct meta-analysis for stent-related inflammation. (A) cholangitis, (B) cholecystitis, and (C) pancreatitis. Table S1: Network estimates of the palliative bile duct stent in terms of recurrent biliary obstruction and adverse events. Table S2: Incidence of recurrent biliary obstruction and adverse events according to the stent type.

Author Contributions: Conceptualization, C.H.P. and S.W.P.; formal analysis, C.H.P., S.W.P. and J.H.J.; interpretation, C.H.P., S.W.P., J.H.J., E.S.J., J.H.K. and D.H.P.; writing—original draft preparation, C.H.P. and S.W.P.; writing-review and editing, C.H.P. and S.W.P. All authors have read and agreed to the published version of the manuscript.

Funding: This research received no external funding.

Institutional Review Board Statement: Ethical review and approval were waived for this study because this is a meta-analysis based on the previously published studies.

Informed Consent Statement: Not applicable.

Data Availability Statement: All relevant data are included in the study and supplementary information.

Conflicts of Interest: The authors declare no conflict of interest.

\section{Appendix A. Detailed Search Strategy}

MEDLINE (Pubmed)

(bile duct[Title/Abstract] OR biliary[Title/Abstract] OR cholangiocarcinoma[Title/ Abstract] OR cholangiocarcinomas[Title/Abstract] OR pancreatic[Title/Abstract] OR pancreas[Title/Abstract]) AND (obstruction[Title/Abstract] OR obstructive[Title/Abstract] OR stricture[Title/Abstract] OR strictures[Title/Abstract] OR jaundice[Title/Abstract] OR cholestasis[Title/Abstract]) AND (nonresectable[Title/Abstract] OR unresectable[Title/Abstract] OR palliation[Title/Abstract] OR palliative[Title/Abstract] OR palliating[Title/Abstract] OR inoperable[Title/Abstract]) AND (stent[Title/Abstract] OR stents[Title/Abstract] OR stenting[Title/Abstract] OR endoprostheses[Title/Abstract]) AND (Randomized Controlled Trial[Publication Type] OR random*[Title/Abstract]) AND (“1990/01/01"[DatePublication]: “2020/01/15"[Date-Publication])

EMBASE (Ovid) 
1: (('bile duct' or biliary or cholangiocarcinoma or cholangiocarcinomas or pancreatic or pancreas) and (obstruction or obstructive or stricture or strictures or jaundice or cholestasis) and (nonresectable or unresectable or palliation or palliative or palliating or inoperable) and (stent or stents or stenting or endoprostheses) and random*).ab, ti.

2: Limit 1 to $(\mathrm{yr}=$ "1990 -Current")

Cochrane library

\#1: 'bile duct' or biliary or cholangiocarcinoma or cholangiocarcinomas or pancreatic or pancreas

\#2: obstruction or obstructive or stricture or strictures or jaundice or cholestasis

\#3: nonresectable or unresectable or palliation or palliative or palliating or inoperable

\#4: stent or stents or stenting or endoprostheses

\#5: random*

\#6: \#1 and \#2 and \#3 and \#4 and \#5 (with Publication Year from 1990 to 2020, in Trials)

\section{References}

1. Artifon, E.L.A.; Sakai, P.; Cunha, J.E.M.; Dupont, A.; Filho, F.M.; Hondo, F.Y.; Ishioka, S.; Raju, G.S. Surgery or endoscopy for palliation of biliary obstruction due to metastatic pancreatic cancer. Am. J. Gastroenterol. 2006, 101, 2031-2037. [CrossRef] [PubMed]

2. Soehendra, N.; Reynders-Frederix, V. Palliative bile duct drainage-A new endoscopic method of introducing a transpapillary drain. Endoscopy 1980, 12, 8-11. [CrossRef] [PubMed]

3. Inamdar, S.; Slattery, E.; Bhalla, R.; Sejpal, D.V.; Trindade, A.J. Comparison of adverse events for endoscopic vs percutaneous biliary drainage in the treatment of malignant biliary tract obstruction in an inpatient national cohort. JAMA Oncol. 2016, 2, 112-117. [CrossRef] [PubMed]

4. Walta, D.C.; Fausel, C.S.; Brant, B. Endoscopic biliary stents and obstructive jaundice. Am. J. Surg. 1987, 153, 444-447. [CrossRef]

5. A Almadi, M.; Barkun, A.; Martel, M. Plastic vs. self-expandable metal stents for palliation in malignant biliary obstruction: A series of meta-analyses. Am. J. Gastroenterol. 2017, 112, 260-273. [CrossRef] [PubMed]

6. Isayama, H.; Hamada, T.; Yasuda, I.; Itoi, T.; Ryozawa, S.; Nakai, Y.; Kogure, H.; Koike, K. TOKYO criteria 2014 for transpapillary biliary stenting. Dig. Endosc. 2015, 27, 259-264. [CrossRef] [PubMed]

7. Sawas, T.; Al Halabi, S.; Parsi, M.A.; Vargo, J. Self-expandable metal stents versus plastic stents for malignant biliary obstruction: A meta-analysis. Gastrointest. Endosc. 2015, 82, 256-267. [CrossRef] [PubMed]

8. Walter, D.; Van Boeckel, P.G.A.; Groenen, M.J.; Weusten, B.L.A.M.; Witteman, B.J.; Tan, G.; Brink, M.A.; Nicolai, J.; Tan, A.C.I.T.L.; Alderliesten, J.; et al. Cost efficacy of metal stents for palliation of extrahepatic bile duct obstruction in a randomized controlled trial. Gastroenterology 2015, 149, 130-138. [CrossRef]

9. Saleem, A.; Leggett, C.L.; Murad, M.H.; Baron, T.H. Meta-analysis of randomized trials comparing the patency of covered and uncovered self-expandable metal stents for palliation of distal malignant bile duct obstruction. Gastrointest. Endosc. 2011, 74, 321-327. [CrossRef]

10. Moole, H.; Bechtold, M.L.; Cashman, M.; Volmar, F.H.; Dhillon, S.; Forcione, D.; Taneja, D.; Puli, S.R. Covered versus uncovered self-expandable metal stents for malignant biliary strictures: A meta-analysis and systematic review. Indian J. Gastroenterol. 2016, 35, 323-330. [CrossRef]

11. Moher, D.; Liberati, A.; Tetzlaff, J.; Altman, D.G. Preferred reporting items for systematic reviews and meta-analyses: The PRISMA statement. Ann. Intern. Med. 2009, 151, 264-269. [CrossRef] [PubMed]

12. Jansen, J.P.; Fleurence, R.; Devine, B.; Itzler, R.; Barrett, A.; Hawkins, N.; Lee, K.; Boersma, C.; Annemans, L.; Cappelleri, J.C.; et al. Interpreting indirect treatment comparisons and network meta-analysis for health-care decision making: Report of the ISPOR task force on indirect treatment comparisons good research practices: Part 1. Value Health 2011, 14, 417-428. [CrossRef] [PubMed]

13. Higgins, J.P.T.; Thompson, S.G.; Deeks, J.J.; Altman, D.G. Measuring inconsistency in meta-analyses. BMJ 2003, 327, 557-560. [CrossRef] [PubMed]

14. Begg, C.B.; Mazumdar, M. Operating characteristics of a rank correlation test for publication bias. Biometrics 1994, 50, 1088. [CrossRef]

15. Easterbrook, P.; Gopalan, R.; Berlin, J.; Matthews, D. Publication bias in clinical research. Lancet 1991, 337, 867-872. [CrossRef]

16. Chandler, J.; Cumpston, M.; Li, T.; Page, M.J.; Welch, V. Cochrane Handbook for Systematic Reviewws of Interventions; John Wiley \& Sons: Hoboken, NJ, USA, 2011.

17. Rücker, G. Network meta-analysis, electrical networks and graph theory. Res. Synth. Methods 2012, 3, 312-324. [CrossRef]

18. Rücker, G.; Schwarzer, G. Ranking treatments in frequentist network meta-analysis works without resampling methods. $B M C$ Med Res. Methodol. 2015, 15, 1-9. [CrossRef]

19. Neupane, B.; Richer, D.; Bonner, A.J.; Kibret, T.; Beyene, J. Network meta-analysis using R: A review of currently available automated packages. PLoS ONE 2014, 9, e115065. [CrossRef]

20. Davids, P.; Groen, A.K.; Rauws, E.; Tytgat, G.; Huibregtse, K. Randomised trial of self-expanding metal stents versus polyethylene stents for distal malignant biliary obstruction. Lancet 1992, 340, 1488-1492. [CrossRef] 
21. Knyrim, K.; Wagner, H.J.; Pausch, J.; Vakil, N. A prospective, randomized, controlled trial of metal stents for malignant obstruction of the common bile duct. Endoscopy 1993, 25, 207-212. [CrossRef]

22. Prat, F.; Chapat, O.; Ducot, B.; Ponchon, T.; Pelletier, G.; Fritsch, J.; Choury, A.D.; Buffet, C. A randomized trial of endoscopic drainage methods for inoperable malignant strictures of the common bile duct. Gastrointest. Endosc. 1998, 47, 1-7. [CrossRef]

23. Kaassis, M.; Boyer, J.; Dumas, R.; Ponchon, T.; Coumaros, D.; Delcenserie, R.; Canard, J.-M.; Fritsch, J.; Rey, J.-F.; Burtin, P.; et al. Plastic or metal stents for malignant stricture of the common bile duct? Results of a randomized prospective study. Gastrointest. Endosc. 2003, 57, 178-182. [CrossRef] [PubMed]

24. Isayama, H.; Komatsu, Y.; Tsujino, T.; Sasahira, N.; Hirano, K.; Toda, N.; Nakai, Y.; Yamamoto, N.; Tada, M.; Yoshida, H.; et al. A prospective randomised study of "covered" versus "uncovered" diamond stents for the management of distal malignant biliary obstruction. Gut 2004, 53, 729-734. [CrossRef] [PubMed]

25. Katsinelos, P.; Paikos, D.; Kountouras, J.; Chatzimavroudis, G.; Paroutoglou, G.; Moschos, I.; Gatopoulou, A.; Beltsis, A.; Zavos, C.; Papaziogas, B.; et al. Tannenbaum and metal stents in the palliative treatment of malignant distal bile duct obstruction: A comparative study of patency and cost effectiveness. Surg. Endosc. 2006, 20, 1587-1593. [CrossRef] [PubMed]

26. Söderlund, C.; Linder, S. Covered metal versus plastic stents for malignant common bile duct stenosis: A prospective, randomized, controlled trial. Gastrointest. Endosc. 2006, 63, 986-995. [CrossRef]

27. Krokidis, M.E.; Fanelli, F.; Orgera, G.; Bezzi, M.; Passariello, R.; Hatzidakis, A. Percutaneous treatment of malignant jaundice due to extrahepatic cholangiocarcinoma: Covered viabil stent versus uncovered wallstents. Cardiovasc. Interv. Radiol. 2010, 33, 97-106. [CrossRef]

28. Kullman, E.; Frozanpor, F.; Söderlund, C.; Linder, S.; Sandström, P.; Lindhoff-Larsson, A.; Toth, E.; Lindell, G.; Jonas, E.; Freedman, J.; et al. Covered versus uncovered self-expandable nitinol stents in the palliative treatment of malignant distal biliary obstruction: Results from a randomized, multicenter study. Gastrointest. Endosc. 2010, 72, 915-923. [CrossRef]

29. Telford, J.J.; Carr-Locke, D.L.; Baron, T.H.; Poneros, J.M.; Bounds, B.C.; Kelsey, P.B.; Schapiro, R.H.; Huang, C.S.; Lichtenstein, D.R.; Jacobson, B.C.; et al. A randomized trial comparing uncovered and partially covered self-expandable metal stents in the palliation of distal malignant biliary obstruction. Gastrointest. Endosc. 2010, 72, 907-914. [CrossRef]

30. Isayama, H.; Yasuda, I.; Ryozawa, S.; Maguchi, H.; Igarashi, Y.; Matsuyama, Y.; Katanuma, A.; Hasebe, O.; Irisawa, A.; Itoi, T.; et al. Results of a japanese multicenter, randomized trial of endoscopic stenting for non-resectable pancreatic head cancer (JM-test): Covered wallstent versus doublelayer stent. Dig. Endosc. 2011, 23, 310-315. [CrossRef]

31. Krokidis, M.; Fanelli, F.; Orgera, G.; Tsetis, D.; Mouzas, I.; Bezzi, M.; Kouroumalis, E.; Pasariello, R.; Hatzidakis, A. Percutaneous palliation of pancreatic head cancer: Randomized comparison of ePTFE/FEP-covered versus uncovered nitinol biliary stents Cardiovasc. Interv. Radiol. 2011, 34, 352-361. [CrossRef]

32. Kitano, M.; Yamashita, Y.; Tanaka, K.; Konishi, H.; Yazumi, S.; Nakai, Y.; Nishiyama, O.; Uehara, H.; Mitoro, A.; Sanuki, T.; et al. Covered self-expandable metal stents with an anti-migration system improve patency duration without increased complications compared with uncovered stents for distal biliary obstruction caused by pancreatic carcinoma: A randomized multicenter trial. Am. J. Gastroenterol. 2013, 108, 1713-1722. [CrossRef] [PubMed]

33. Moses, P.L.; AlNaamani, K.M.; Barkun, A.; Gordon, S.R.; Mitty, R.D.; Branch, M.S.; E Kowalski, T.; Martel, M.; Adam, V. Randomized trial in malignant biliary obstruction: Plastic vs partially covered metal stents. World J. Gastroenterol. 2013, 19, 8638-8646. [CrossRef] [PubMed]

34. Ung, K.A.; Stotzer, P.-O.; Nilsson, Å.; Gustavsson, M.-L.; Johnsson, E. Covered and uncovered self-expandable metallic Hanarostents are equally efficacious in the drainage of extrahepatic malignant strictures: Results of a double-blind randomized study. Scand. J. Gastroenterol. 2012, 48, 459-465. [CrossRef] [PubMed]

35. Lee, S.J.; Kim, M.-D.; Lee, M.S.; Kim, I.J.; Park, S.I.; Won, J.Y.; Lee, D.Y. Comparison of the efficacy of covered versus uncovered metallic stents in treating inoperable malignant common bile duct obstruction: A randomized trial. J. Vasc. Interv. Radiol. 2014, 25, 1912-1920. [CrossRef]

36. Yang, M.J.; Kim, J.H.; Yoo, B.M.; Hwang, J.C.; Yoo, J.H.; Lee, K.S.; Kang, J.K.; Kim, S.S.; Lim, S.G.; Shin, S.J.; et al. Partially covered versus uncovered self-expandable nitinol stents with anti-migration properties for the palliation of malignant distal biliary obstruction: A randomized controlled trial. Scand. J. Gastroenterol. 2015, 50, 1490-1499. [CrossRef]

37. Bernon, M.M.; Shaw, J.; Burmeister, S.; Chinnery, G.; Hofmeyr, S.; Kloppers, J.C.; Jonas, E.; Krige, J.E.J. Distal malignant biliary obstruction: A prospective randomised trial comparing plastic and uncovered self-expanding metal stents in the palliation of symptomatic jaundice. South Afr. J. Surg. 2018, 56, 30-34. [CrossRef]

38. Conio, M.; Mangiavillano, B.; Caruso, A.; Filiberti, R.A.; Baron, T.H.; De Luca, L.; Signorelli, S.; Crespi, M.; Marini, M.; Ravelli, P.; et al. Covered versus uncovered self-expandable metal stent for palliation of primary malignant extrahepatic biliary strictures: A randomized multicenter study. Gastrointest. Endosc. 2018, 88, 283-291. [CrossRef]

39. Dhondt, E.; Vanlangenhove, P.; De Man, M.; Huyck, L.; Defreyne, L. No advantage of expanded polytetrafluoroethylene and fluorinated ethylene propylene-covered stents over uncovered nitinol stents for percutaneous palliation of malignant infrahilar biliary obstruction: Results of a single-center prospective randomized trial. J. Vasc. Interv. Radiol. 2020, 31, 82-92.

40. Perdue, D.; Freeman, M.; DiSario, J.; Nelson, D.; Fennerty, M.; Lee, J.; Overby, C.; Ryan, M.; Bochna, G.; Snady, H.; et al. Plastic versus self-expanding metallic stents for malignant hilar biliary obstruction: A prospective multicenter observational cohort study. J. Clin. Gastroenterol. 2008, 42, 1040-1046. [CrossRef] 
41. Raju, R.P.; Jaganmohan, S.R.; Ross, W.A.; Davila, M.L.; Javle, M.; Raju, G.S. Optimum palliation of inoperable hilar cholangiocarcinoma: Comparative assessment of the efficacy of plastic and self-expanding metal stents. Dig. Dis. Sci. 2011, 56, 1557-1564. [CrossRef]

42. Speer, A.G.; Cotton, P.B.; Macrae, K.D. Endoscopic management of malignant biliary obstruction: Stents of 10 French gauge are preferable to stents of 8 French gauge. Gastrointest. Endosc. 1988, 34, 412-417. [CrossRef]

43. Luigiano, C.; Ferrara, F.; Cennamo, V.; Fabbri, C.; Bassi, M.; Ghersi, S.; Consolo, P.; Morace, C.; Polifemo, A.M.; Billi, P.; et al. A comparison of uncovered metal stents for the palliation of patients with malignant biliary obstruction: Nitinol vs. stainless steel. Dig. Liver Dis. 2012, 44, 128-133. [CrossRef] [PubMed]

44. Ho, H.; Mahajan, A.; Gosain, S.; Jain, A.; Brock, A.; Rehan, M.E.; Ellen, K.; Shami, V.M.; Kahaleh, M. Management of complications associated with partially covered biliary metal stents. Dig. Dis. Sci. 2009, 55, 516-522. [CrossRef] [PubMed]

45. Weber, A.; Mittermeyer, T.; Wagenpfeil, S.; Schmid, R.M.; Prinz, C. Self-expanding metal stents versus polyethylene stents for palliative treatment in patients with advanced pancreatic cancer. Pancreas 2009, 38, e7-e12. [CrossRef] [PubMed]

46. Coté, G.A.; Kumar, N.; Ansstas, M.; Edmundowicz, S.A.; Jonnalagadda, S.; Mullady, D.K.; Azar, R.R. Risk of post-ERCP pancreatitis with placement of self-expandable metallic stents. Gastrointest. Endosc. 2010, 72, 748-754. [CrossRef] [PubMed]

47. Nakai, Y.; Isayama, H.; Togawa, O.; Kogure, H.; Tsujino, T.; Yagioka, H.; Yashima, Y.; Sasaki, T.; Ito, Y.; Matsubara, S.; et al. New method of covered wallstents for distal malignant biliary obstruction to reduce early stent-related complications based on characteristics. Dig. Endosc. 2010, 23, 49-55. [CrossRef]

48. Sogabe, Y.; Kodama, Y.; Honjo, H.; Aoyama, I.; Muramoto, Y.; Koga, E.; Yanaidani, T.; Kawai, M.; Yoshikawa, T.; Matsumoto, S.; et al. Tumor invasion to the arteries feeding the gallbladder as a novel risk factor for cholecystitis after metallic stent placement in distal malignant biliary obstruction. Dig. Endosc. 2018, 30, 380-387. [CrossRef]

49. Tringali, A.; Hassan, C.; Rota, M.; Rossi, M.; Mutignani, M.; Aabakken, L. Covered vs. uncovered self-expandable metal stents for malignant distal biliary strictures: A systematic review and meta-analysis. Endoscopy 2018, 50, 631-641.

50. Kawakubo, K.; Isayama, H.; Nakai, Y.; Togawa, O.; Sasahira, N.; Kogure, H.; Sasaki, T.; Matsubara, S.; Yamamoto, N.; Hirano, K.; et al. Risk factors for pancreatitis following transpapillary self-expandable metal stent placement. Surg. Endosc. 2011, 26, 771-776. [CrossRef]

51. Nakai, Y.; Isayama, H.; Mukai, T.; Itoi, T.; Maetani, I.; Kawakami, H.; Yasuda, I.; Maguchi, H.; Ryozawa, S.; Hanada, K.; et al. Impact of anticancer treatment on recurrent obstruction in covered metallic stents for malignant biliary obstruction. J. Gastroenterol. 2013, 48, 1293-1299. [CrossRef]

52. Park, D.H.; Lee, S.S.; Lee, T.H.; Ryu, C.H.; Kim, H.J.; Seo, D.-W.; Park, S.-H.; Lee, S.-K.; Kim, M.-H.; Kim, S.-J.; et al. Anchoring flap versus flared end, fully covered self-expandable metal stents to prevent migration in patients with benign biliary strictures: A multicenter, prospective, comparative pilot study (with videos). Gastrointest. Endosc. 2011, 73, 64-70. [CrossRef] [PubMed] 